\title{
Solubility of 7-Chloro-2-methylamino-5-phenyl-3H-1,4-benzodiazepine-4-oxide, 7-Chloro-1,3-dihydro-1-methyl-5-phenyl-2H-1,4-benzodiazepin-2-one, and 7-Chloro-5-(2-chlorophenyl)-3-hydroxy-1,3-dihydro-1,4-benzodiazepin-2-one in (Propane-1,2-diol + Water) at a Temperature of 303.2 K
}

\author{
Abolghasem Jouyban, ${ }^{\dagger \dagger}$ Javad Shokri, ${ }^{\ddagger}$ Mohammad Barzegar-Jalali, ${ }^{\ddagger}$ Davoud Hassanzadeh, ${ }^{\S}$ William E. Acree, Jr.," \\ Taravat Ghafourian, ${ }^{\perp}$ and Ali Nokhodchi ${ }^{\perp}$
}

Faculty of Pharmacy and Drug Applied Research Center, Biotechnology Research Center, and Research Center for Pharmaceutical Nanotechnology, Tabriz University (Medical Sciences), Tabriz 51664, Iran, Department of Chemistry, University of North Texas, Denton, Texas 76203-5070, and Medway School of Pharmacy, Universities of Kent and Greenwich, Kent ME4 4TB, United Kingdom

\begin{abstract}
Experimental solubilities of 7-chloro-2-methylamino-5-phenyl-3H-1,4-benzodiazepine-4-oxide (chlordiazepoxide), 7-chloro-1,3-dihydro-1-methyl-5-phenyl-2H-1,4-benzodiazepin-2-one (diazepam), and 7-chloro5-(2-chlorophenyl)-3-hydroxy-1,3-dihydro-1,4-benzodiazepin-2-one (lorazepam) in (propane-1,2-diol + water) at $T=303.2 \mathrm{~K}$ were reported. The solubility of drugs increased with the addition of propane-1,2-diol and reached the maximum values in pure propane-1,2-diol. The Jouyban-Acree model was used to fit the experimental data, and the solubilities were reproduced using a previously trained version of the Jouyban-Acree model and the solubility data in monosolvents in which the overall mean relative deviations (OMRDs) of the back-calculated and predicted values with the corresponding experimental data were 4.2 $\%$ and $10.7 \%$. The solubilities of the three drugs were also predicted using a trained version of the log-linear model of Yalkowsky, and the OMRD was $21.1 \%$.
\end{abstract}

\section{Introduction}

Propane-1,2-diol (known as propylene glycol in the pharmaceutical industry) is a common and safe cosolvent to be used in pharmaceutical formulations. Its solubilization power is reasonably high, and in many drug formulations the concentration of propane-1,2-diol is lower than $50 \%$. Propane-1,2-diol is a stable and low toxic pharmaceutical cosolvent which is used as a single or in combination with other cosolvents such as ethanol or polyethylene glycols in oral and parenteral pharmaceutical formulations of poorly soluble drugs. ${ }^{1,2}$

The solubility of drugs in propane-1,2-diol + water mixtures is essential information in drug discovery and development studies. The data could be used in recrystallization of drugs and excipients (particle engineering) and also in formulation processes. In addition to the solubilization effects of propane1,2-diol, it has a stabilization effect against hydrolytic reactions as well, and it could increase the stability of benzodiazepines in the aqueous formulations. ${ }^{3}$

Despite experimental effort for determination of the solubility of drugs in water + cosolvent mixtures, a number of mathematical models have been presented for predicting the solubility of drugs in these mixtures which are reviewed in a recent work. ${ }^{4}$ Of the numerous models developed in recent years, the

* To whom correspondence should be addressed. E-mail: ajouyban@ hotmail.com. Fax: +98 4113363231 .

Faculty of Pharmacy and Drug Applied Research Center, Tabriz University (Medical Sciences).

Biotechnology Research Center, Tabriz University (Medical Sciences).

$\S$ Research Center for Pharmaceutical Nanotechnology, Tabriz University (Medical Sciences).

"University of North Texas.

${ }^{\perp}$ Medway School of Pharmacy, Universities of Kent and Greenwich.
Jouyban-Acree model is perhaps one of the more versatile models. The model provides very accurate mathematical descriptions for how the solute solubility varies with both temperature and solvent composition. The model is

$$
\begin{aligned}
\log C_{m, T}^{\mathrm{Sat}}=\phi_{1} \log C_{1, T}^{\mathrm{Sat}}+\phi_{2} \log C_{2, T}^{\mathrm{Sat}}+ & \\
& \frac{\phi_{1} \phi_{2}}{T} \sum_{i=0}^{2} J_{i}\left(\phi_{1}-\phi_{2}\right)^{i}
\end{aligned}
$$

where $C_{m, T}^{\text {Sat }}$ is the solute solubility in the binary solvent mixtures at temperature $T ; \phi_{1}$ and $\phi_{2}$ are the volume fractions of the solvents 1 (propane-1,2-diol) and 2 (water) in the absence of the solute; $C_{1, T}^{\mathrm{Sat}}$ and $C_{2, T}^{\mathrm{Sat}}$ denote the solubility of the solute in the neat solvents 1 and 2 , respectively; and $J_{i}$ are the constants of the model computed by a regression analysis. ${ }^{4}$ The existence of these model constants which require a number of solubility data in water-cosolvent mixtures for the training process is a limitation for the model when the solubility predictions are the goal of the computations in early drug discovery studies. This limitation could be resolved using a trained version of the model for a given water-cosolvent mixture. The trained version of the Jouyban-Acree model for prediction of drug solubility in (propane-1,2-diol + water) at temperature $T$ is ${ }^{5}$

$$
\begin{array}{r}
\log C_{m, T}^{\mathrm{Sat}}=\phi_{1} \log C_{1, T}^{\mathrm{Sat}}+\phi_{2} \log C_{2, T}^{\mathrm{Sat}}+\frac{37.03 \phi_{1} \phi_{2}}{T}+ \\
\frac{319.49 \phi_{1} \phi_{2}\left(\phi_{1}-\phi_{2}\right)}{T}
\end{array}
$$

Equation 2 is only applicable for solubility prediction of drugs in (propane-1,2-diol + water), and the effect of the drug's functional groups on the solubility was ignored. Equation 2 
Table 1. Absorbance $A$ and Calibration Coefficient $C$ of the Spectrometer at Wavelength $\lambda$ and Molarity Range $C$ with Drugs in Methanol

\begin{tabular}{|c|c|c|c|c|c|}
\hline & $\lambda$ & $\varepsilon$ & $C$ & & calibration curve \\
\hline drug & $\mathrm{nm}$ & $\mathrm{dm}^{3} \cdot \mathrm{mol}^{-1} \cdot \mathrm{cm}^{-1}$ & $\mathrm{~mol} \cdot \mathrm{dm}^{-3}$ & $\begin{array}{l}\text { correlation } \\
\text { coefficient }\end{array}$ & $\begin{array}{l}\text { (A: relative } \\
\text { absorbance) }\end{array}$ \\
\hline chlordiazepoxide & 234 & 30167 to 31568 & $2.25 \cdot 10^{-6}$ to $3.34 \cdot 10^{-5}$ & 0.999 & $C=3.326 \cdot 10^{-5} A-1.125 \cdot 10^{-7}$ \\
\hline lorazepam & 231 & 37980 to 38924 & $2.10 \cdot 10^{-5}$ to $3.11 \cdot 10^{-5}$ & 0.999 & $C=2.638 \cdot 10^{-5} A-5.615 \cdot 10^{-8}$ \\
\hline
\end{tabular}

Table 2. Experimental Solubilities of Chlordiazepoxide, Diazepam, and Lorazepam at Different Volume Fractions of Propane-1,2-diol $\left(\phi_{1}\right)$ in Propane-1,2-diol (1) + Water (2) Mixtures at 303.2 K and Density $\rho$ of the Saturated Solutions

\begin{tabular}{|c|c|c|c|c|c|c|}
\hline \multirow[b]{2}{*}{$\varphi_{1}$} & \multicolumn{3}{|c|}{$C_{m, T}^{\mathrm{Sat}} / \mathrm{mol} \cdot \mathrm{dm}^{-3}$} & \multicolumn{3}{|c|}{$\rho / \mathrm{g} \cdot \mathrm{cm}^{-3}$} \\
\hline & chlordiazepoxide & diazepam & lorazepam & chlordiazepoxide & diazepam & lorazepam \\
\hline 0.000 & 0.000354 & 0.000192 & 0.000193 & 1.000 & 0.999 & 1.001 \\
\hline 0.100 & 0.000590 & 0.000297 & 0.000290 & 1.004 & 1.010 & 1.010 \\
\hline 0.200 & 0.000800 & 0.000492 & 0.000480 & 1.009 & 1.012 & 1.016 \\
\hline 0.300 & 0.001270 & 0.000928 & 0.001080 & 1.023 & 1.021 & 1.029 \\
\hline 0.400 & 0.002590 & 0.001910 & 0.002580 & 1.028 & 1.027 & 1.034 \\
\hline 0.500 & 0.004270 & 0.004410 & 0.004500 & 1.031 & 1.039 & 1.034 \\
\hline 0.600 & 0.009180 & 0.009620 & 0.007810 & 1.035 & 1.040 & 1.045 \\
\hline 0.700 & 0.013500 & 0.016800 & 0.013100 & 1.047 & 1.044 & 1.049 \\
\hline 0.800 & 0.022700 & 0.030800 & 0.021600 & 1.043 & 1.047 & 1.053 \\
\hline 0.900 & 0.039500 & 0.048900 & 0.032700 & 1.041 & 1.049 & 1.048 \\
\hline 1.000 & 0.065900 & 0.069600 & 0.064800 & 1.042 & 1.043 & 1.045 \\
\hline
\end{tabular}

Table 3. Numerical Values of Adjusted Parameters of Equation 1 for Each Solute and the Mean Relative Deviation (100•MRD) for the Predicted Solubilities of Drugs in Propane-1,2-diol (1) + Water (2) Mixtures Using Various Numerical Analyses and Their Overall Values

\begin{tabular}{lrccrrr}
\hline \multicolumn{1}{c}{ drug } & $J_{0}$ & $J_{1}$ & $J_{2}$ & method I & method II & method III \\
\hline chlordiazepoxide & -51.779 & 153.060 & $-^{a}$ & 5.8 & 14.0 & 9.4 \\
diazepam & 72.410 & 421.197 & $-^{a}$ & 3.4 & 5.5 & 22.3 \\
lorazepam & 139.017 & 130.617 & -571.385 & 3.6 & 12.5 & 31.5 \\
& & & overall: & 4.3 & 10.7
\end{tabular}

${ }^{a}$ Not significant.

enables one to predict the solubilities of any crystalline nonelectrolyte solute in (propane-1,2-diol + water) provided that the solute's solubility in the two monosolvents propane1,2-diol and water is known.

An alternative prediction method is the trained version of the $\log$-linear model of Yalkowsky ${ }^{6,7}$ which is expressed by

$$
\log C_{m, T}^{\mathrm{Sat}}=\log C_{2, T}^{\mathrm{Sat}}+(0.37+0.78 \log P) \phi_{1}
$$

where $\log P$ is the logarithm of the drug's partition coefficient. ${ }^{6}$ The numerical values of $\log P$ for chlordiazepoxide, diazepam, and lorazepam employed in this work were 2.44, 2.70, and 2.40, respectively. ${ }^{8}$

Experimental solubility of diazepam in (propane-1,2-diol + water) at a temperature of $298.15 \mathrm{~K}$ was reported in a previous work. ${ }^{9}$ In this work, the experimental solubility of chlordiazepoxide, diazepam, and lorazepam in (propane-1,2-diol + water) at $T=303.2 \mathrm{~K}$ is reported. There were no published data on the solubility of these drugs in (propane-1,2-diol + water) at $T$ $=303.2 \mathrm{~K}$. In addition, we illustrate the applicability of the Jouyban-Acree model to the measured drug solubility data and assess the prediction capability of the above-mentioned trained models given above for predicting the solubility of drugs in (propane-1,2-diol + water).

\section{Experimental Method}

Materials. Chlordiazepoxide (0.997 in mass fraction) or 7-chloro-2-methylamino-5-phenyl-3H-1,4-benzodiazepine-4oxide (CAS \# 58-25-3), diazepam (0.997 in mass fraction), or 7-chloro-1,3-dihydro-1-methyl-5-phenyl-2H-1,4-benzodiazepin2-one (CAS \# 439-14-5) and lorazepam (0.998 in mass fraction) or 7-chloro-5-(2-chlorophenyl)-3-hydroxy-1,3-dihydro-1,4-benzodiazepin-2-one (CAS \# 846-49-1) were purchased from Loghman pharmaceutical company (Iran). Propane-1,2-diol
(0.995 in mass fraction) was purchased from Merck (Germany); methanol (0.998 in mass fraction) was obtained from Caledon (Canada); and double distilled water was used for preparation of the solutions.

Apparatus and Procedures. The binary solvent mixtures were prepared by mixing the appropriate volumes of the solvents with the accuracy of 0.001 volume fraction. The solubility of benzodiazepines in (propane-1,2-diol + water) was determined by equilibrating excess amounts of the solids at a temperature of $303.2 \mathrm{~K}$ using a shaker (Behdad, Tehran, Iran) placed in an incubator equipped with a temperature-controlling system maintained constant to within $\pm 0.2 \mathrm{~K}$. After a sufficient length of time $(>24 \mathrm{~h})$, the saturated solutions of the drugs were filtered using hydrophilic Durapore filters $(0.45 \mu \mathrm{m}$, Milipore, Ireland), diluted with methanol, and then assayed at (234, 231, and 231) $\mathrm{nm}$, respectively, for chlordiazepoxide, diazepam, and lorazepam using a UV-vis spectrophotometer (Beckman DU-650, Fullerton). The preliminary investigations showed that the filter did not absorb the solutes through the filtration process. Concentrations of the diluted solutions were determined from the calibration curves. Details of calibration curves are shown in Table 1. Each experimental data point represents the average of at least three repetitive experiments with the measured solubilities being reproducible on a relative basis within \pm 6.3 $\%$. Calculated standard relative deviations of solubilities ranged from $1.3 \%$ to $6.3 \%$. The densities of the saturated solutions were determined using a $5 \mathrm{~mL}$ pycnometer with the uncertainty of $\pm(0.005$ to 0.025$) \mathrm{g} \cdot \mathrm{cm}^{-3}$.

Computational Methods. In the numerical analysis of method I, eq 1 was fitted to the experimental solubility data of each drug, and the back-calculated solubilities were used to calculate the accuracy of the fit. In method II, the solubilities of three drugs were predicted using eq 2 employing the experimental 


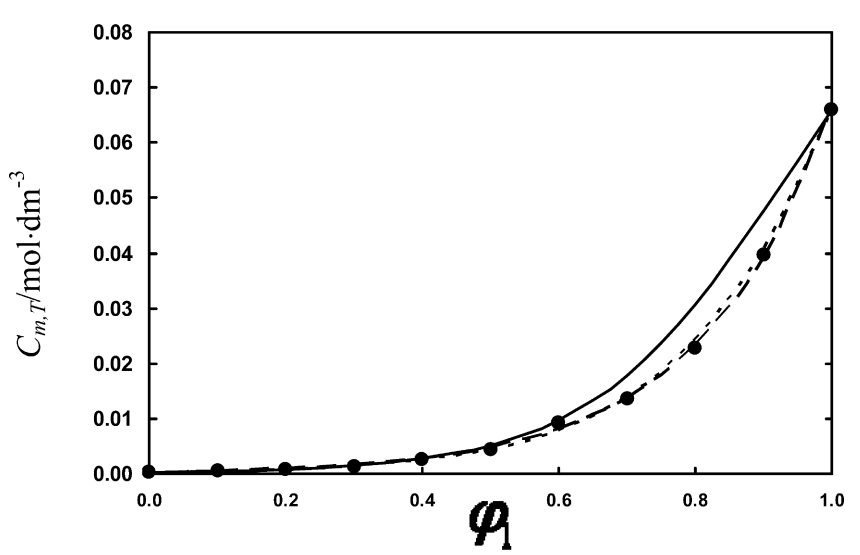

Figure 1. Solubility of chlordiazepoxide $C_{m, T}$ as a function of volume fractions of propane-1,2-diol $\left(\phi_{1}\right)$ in (propane-1,2-diol + water): $\bullet$, experimental; and the computed solubilities using: - - - , method I; - , method II; - - method III.

solubilities of drugs in pure propane-1,2-diol and water. In method III, solubilities of these drugs were predicted using a trained version of the log-linear model (eq 3 ) and their aqueous solubility at a temperature of $303.2 \mathrm{~K}$. The mean relative deviation (MRD) was used to check the accuracy of the prediction methods and is calculated using

$$
\text { MRD }=\frac{1}{N} \sum\left\{\frac{\left|\left(C_{m}^{\text {Sat }}\right)_{\text {pred }}-\left(C_{m}^{\text {Sat }}\right)\right|}{\left(C_{m}^{\text {Sat }}\right)}\right\}
$$

where $N$ is the number of data points in each set. Goodness of the fit to each method was also shown by plotting the predicted and experimental solubilities of the drugs against the volume fraction of propane-1,2-diol.

\section{Results and Discussion}

Table 2 lists the experimental solubilities of chlordiazepoxide, diazepam, and lorazepam in propane-1,2-diol + water mixtures at a temperature of $303.2 \mathrm{~K}$. There is fairly good agreement between aqueous solubility data of diazepam at a temperature of $303.2 \mathrm{~K}$ from this work $\left(0.00019 \mathrm{~mol} \cdot \mathrm{dm}^{-3}\right)$, a published solubility data of diazepam in water at a temperature of 303.15 $\mathrm{K}\left(0.00014 \mathrm{~mol} \cdot \mathrm{dm}^{-3}\right),{ }^{8}$ and a recently reported value for the solubility of diazepam in water at a temperature of $298.15 \mathrm{~K}$ $\left(0.00015 \mathrm{~mol} \cdot \mathrm{dm}^{-3}\right){ }^{9}$ Differences in chemical purities and experimental methodologies can lead to slight differences in measured solubility data. Comparing these three values, we note that our measured value of $0.00019 \mathrm{~mol} \cdot \mathrm{dm}^{-3}$ at a temperature of $303.2 \mathrm{~K}$ is slightly larger than the value of $0.00015 \mathrm{~mol} \cdot \mathrm{dm}^{-3}$ reported by Shayanfar et al. ${ }^{9}$ This is to be expected as diazepam will be more soluble at the higher temperature. The experimental value of $0.00014 \mathrm{~mol} \cdot \mathrm{dm}^{-3}$ is a questionable datum. There is also a parallel shift in solubility data of diazepam at various compositions of (propane-1,2-diol + water) at a temperature of $298.15 \mathrm{~K}^{9}$ and the corresponding data at a temperature of $303.2 \mathrm{~K}$ from this work. The solubility of drugs increased with the addition of propane-1,2-diol and reached the maximum values in pure propane-1,2-diol.

The solubility of the benzodiazepines was predicted using numerical methods I, II, and III. The experimental and predicted solubilities of the drugs versus the volume fraction of propane1,2-diol in the mixtures were plotted in Figures 1, 2, and 3. As shown in the figures, the Jouyban-Acree model fits very well to the experimental solubility data of drugs at all composition

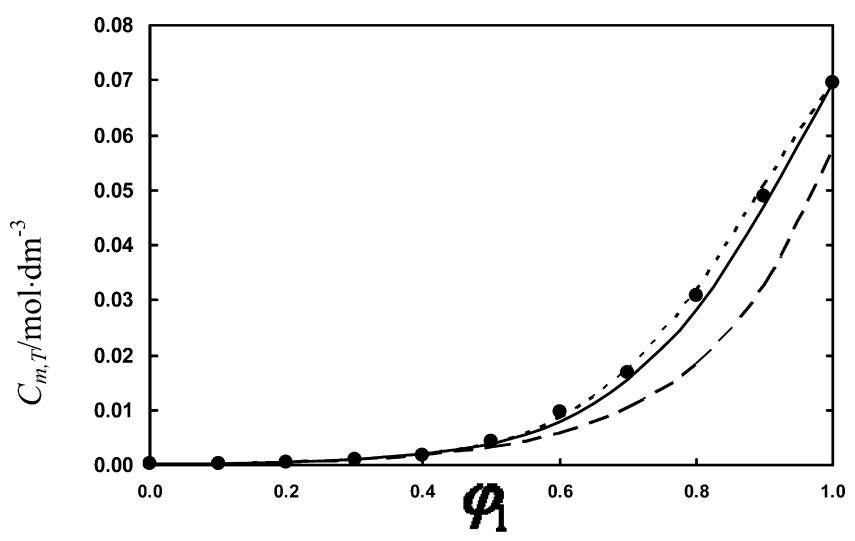

Figure 2. Solubility of diazepam $C_{m, T}$ as a function of volume fractions of propane-1,2-diol $\left(\phi_{1}\right)$ in (propane-1,2-diol + water): $\bullet$, experimental; and the computed solubilities using: - - - -, method I; - , method II; - - , method III.

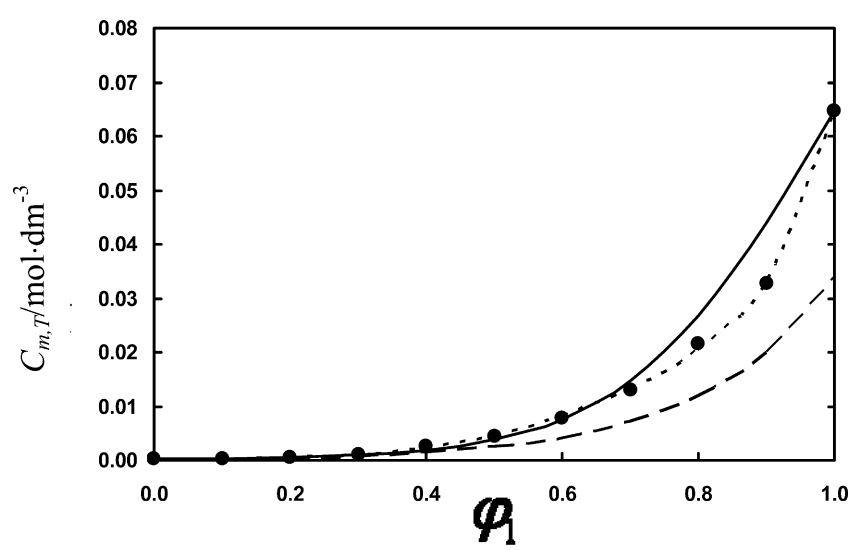

Figure 3. Solubility of lorazepam $C_{m, T}$ as a function of volume fractions of propane-1,2-diol $\left(\phi_{1}\right)$ in (propane-1,2-diol + water): $\bullet$, experimental; and the computed solubilities using: - - -, method I; -, method II; - -, method III.

ranges of propane-1,2-diol. This finding is also supported by small MRD values of the back-calculated and experimental solubility data. The main limitation of eq 1 is that it should be trained for each drug employing a minimum number of experimental data in binary solvents. The predictive version of the model, i.e., eq 2 , predicts the solubility values with reasonable MRD values. The predicted solubilities were compared with the corresponding experimental data, and MRD values were computed and listed in Table 3 . As noticed above, the $\log$-linear model of Yalkowsky is an alternative model requiring the aqueous solubility data and $\log P$ values of the solutes. It provides rough estimated solubility data, and the overall MRD was $21.1 \%$. Generally, the overall MRDs of the Jouyban-Acree model reveal that its trained version for (propane-1,2-diol + water) is a robust model and could be used for prediction purposes within an acceptable error range.

\section{Literature Cited}

(1) Strickley, R. G. Solubilizing Excipients in Oral and Injectable Formulations. Pharm. Res. 2004, 21, 201-230.

(2) Yalkowsky, S. H.; Rubino, J. T. Solubilization by Cosolvents I: Organic Solutes in Propylene Glycol-Water Mixtures. J. Pharm. Sci. 1985, 74, 416-421.

(3) Wells, J. I. Pharmaceutical Preformulation: The Physicochemical Properties of Drug Substances; Ellis Harwood: Chichester, 1988; p 40.

(4) Jouyban, A. Review of the Cosolvency Models for Predicting Solubility of Drugs in Water-Cosolvent Mixtures. J. Pharm. Pharm. Sci. 2008, $11,32-58$. 
(5) Jouyban, A. Prediction of Drug Solubility in Water-Propylene Glycol Mixtures Using Jouyban-Acree Model. Pharmazie 2007, 62, 365-367.

(6) Yalkowsky, S. H.; Roseman, T. Solubilization of Drugs by Cosolvents. Yalkowsky, S. H., Ed.; Techniques of Solubilization of Drugs; Marcel Dekker: New York, 1981; pp 91-134.

(7) Li, A.; Yalkowsky, S. H. Predicting Cosolvency. 1. Solubility Ratio and Solute log Kow. Ind. Eng. Chem. Res. 1998, 37, 4470-4475.

(8) Moffat, A. C. Clarke's Analysis of Drug and Poisons; Pharmaceutical Press: London, 2004.
(9) Shayanfar, A.; Acree, W. E., Jr.; Jouyban, A. Solubility of Lamotrigine, Diazepam, Clonazepam, and Phenobarbital in Propylene Glycol + Water Mixtures at 298.15 K. J. Chem. Eng. Data 2009, 54, 11531157.

Received for review April 6, 2009. Accepted July 21, 2009.

JE900451D 